\title{
The Plume Impingement Contamination II Experiment: Motivation, Design, and Implementation Plan
}

\author{
Forrest E. Lumpkin III, \\ Keith C. Albyn, \\ Thomas L. Farrell, \\ NASA Johnson Space Center \\ Houston, TX
}

The International Space Station (ISS) will have a long service life during which it must be able to serve as a capable platform for a wide variety of scientific investigations. In order to provide this capability, the ISS has, at the system level, a design requirement of no more than 100 Angstroms of contaminant deposition per year from "non-quiescent" sources. Non-quiescent sources include the plumes resulting from the firing of reaction control system (RCS) engines on space vehicles visiting the ISS as well as the engines on the ISS itself. Unfortunately, good general plume contamination models do not yet exist. This is due both to the complexity of the problem, making the analytic approach difficult, and to the difficulty in obtaining empirical measurements of contaminant depositions. To address this lack of flight data, NASA Johnson Space Center is planning to fly an experiment, Plume Impingement Contamination-II, to measure the contamination deposition from the Shuttle Orbiter's primary RCS engines as a function angle from plume centerline. This represents the first direct on-orbit measurement of plume impingement contamination away from the nozzle centerline ever performed, and as such is extremely important in validating mathematical models which will be used to quantify the cumulative plume impingement contamination to the ISS over its lifetime. The paper will elaborate further upon the motivation behind making these measurements as well as present the design and implementation plan of this planned experiment. 